K. OKI, M. TAKASE*, S. MORI, A. SHIOTARI, Y. SUGIMOTO, K. OHARA, T. OKUJIMA,

H. UNO* (EHIME UNIVERSITY, MATSUYAMA AND THE UNIVERSITY OF TOKYO, KASHIWA, JAPAN)

Synthesis, Structures, and Properties of Core-Expanded Azacoronene Analogue: A Twisted $\pi$-System with Two

N-Doped Heptagons

J. Am. Chem. Soc. 2018, 140, 10430-10434.

\section{Lucky Seven-Membered Rings in N-Doped Nonplanar Nanographenes}
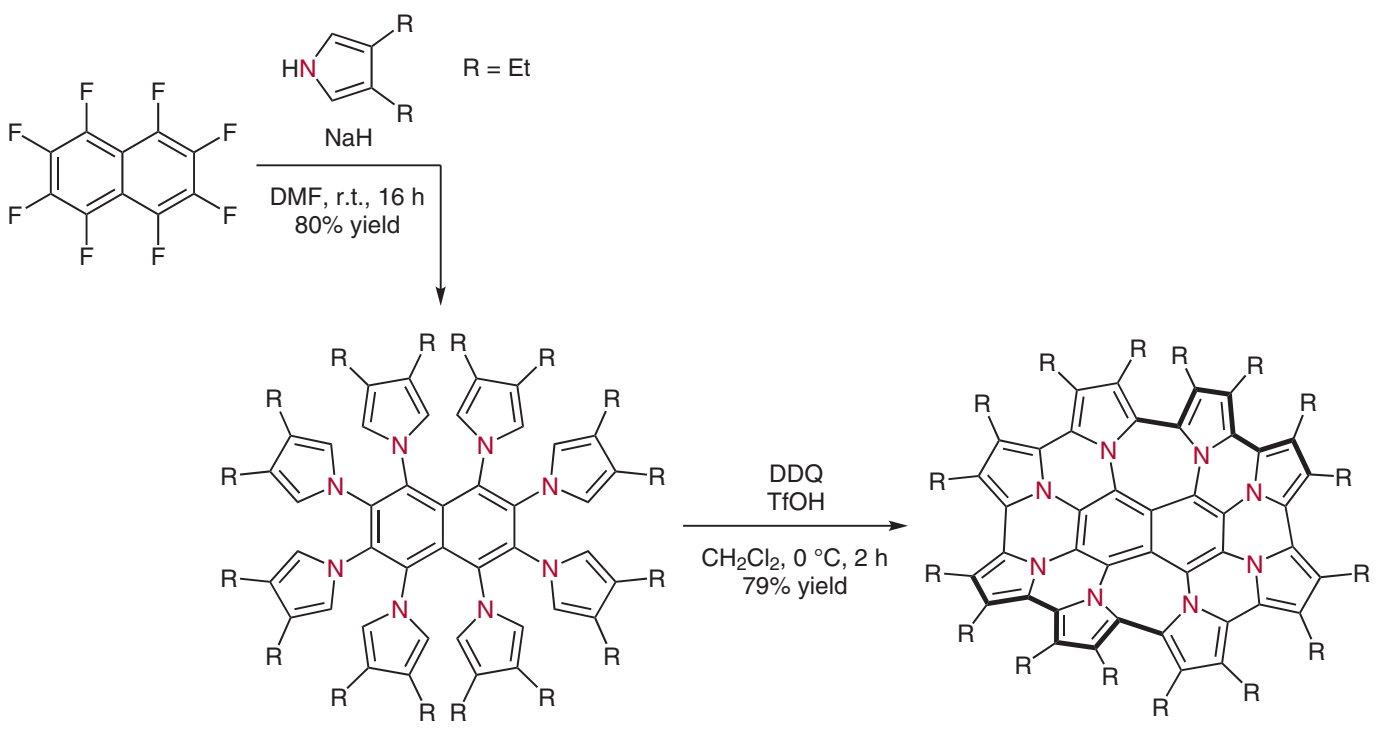

Significance: Nanographenes containing heptagonal rings have received attention because of their unique electronic properties arising from their nonplanarity. Introducing heteroatoms into nanographenes is also known to affect their electronic nature without modifying the structure. However, heteroatom doping in nonplanar nanographenes containing heptagonal rings are not well known. Here, Takase, Uno and co-workers report a rapid two-step synthesis of a core-expanded azacoronene analogue, which possesses two heptagonal rings and eight inner nitrogen atoms.
Comment: This electron-rich azacoronene analogue showed four reversible oxidation peaks. Single crystals of the neutral state and dicationic state could be obtained. From the bond-length alternation, nucleus-independent chemical shift and anisotropy of the induced current density calculations, the authors conclude overall Hückel aromaticity through $30 \pi$ conjugation in the dicationic state, despite being nonplanar.
Category

Synthesis of Materials and

Unnatural Products

\section{Key words}

azaaromatics

electronic materials

oxidation

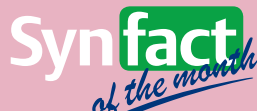

